\title{
Validation of staging systems for gastric cancer
}

\author{
Keishi Yamashita, Shinichi Sakuramoto, Shiroh Kikuchi, Natsuya Katada, Nobuyuki Kobayashi, \\ and MasahiKo WatANABE \\ Department of Surgery, Kitasato University Hospital, 1-15-1 Kitastato, Sagamihara, Kanagawa 228-8555, Japan
}

\begin{abstract}
Background. The two major staging systems for gastric cancer, the Japanese classification of gastric cancer (JCGC) and the International Union Against Cancer (UICC) TNM system, are periodically revised as a consequence of critical validation studies in light of newly accumulated clinical data. This study aimed to validate and improve upon the current versions for a better prognostic stratification of gastric cancer.

Methods. One thousand and ten gastric cancer patients who underwent tumor resection were enrolled at the Kitasato University Hospital for staging validation. According to the JCGC stage, the patients consisted of stage IA $(n=453)$, IB $(n=185)$, II $(n=119)$, IIIA $(n=75)$, IIIB $(n=51)$, and IV $(n=127)$.

Results. Regarding consistency between the JCGC and the UICC system, the results were: for patients in stage IA (100\%), IB (98\%), II (84\%), IIIA (51\%), IIIB (24\%), and IV (64\%). The JCGC system was superior to the UICC system for the prognostic stratification of stage IIIA, IIIB, and IV cancers; we therefore used the JCGC system for prognostic validation according to depth of invasion in cancers of the same stage. Stage II and IIIA cancers were heterogeneous for prognosis according to depth of invasion, and the outstanding difference was found between the muscularis propria (MP) and subserosa (SS), which are both classified as pT2 in the JCGC system. MP cancer represented an earlier property of gastric cancer rather than an advanced one. A proposed novel staging system adjusted for this heterogeneity provided a clearer stratification of prognosis with a homogeneous prognostic distribution within each stage.

Conclusion. Our findings revealed that invasion into the MP has an earlier propensity than expected, and a novel staging system taking this into account may provide a better stratification of prognosis than the current systems.
\end{abstract}

Key words Gastric Cancer · Staging · Validation · MP

Offprint requests to: $\mathrm{M}$. Watanabe

Received: June 12, 2007 / Accepted: May 1, 2008

\section{Introduction}

The incidence and mortality rate of gastric cancer have decreased dramatically over the past several decades [1]; nonetheless, the disease remains a major public health issue as the fourth most common cancer and the second leading cause of cancer death worldwide [2]. Radical surgery in combination with systemic lymph node dissection is the current treatment of choice for gastric cancer [3]. The two major systems used for staging are the Japanese classification of gastric cancer (JCGC), which is currently in its thirteenth edition (corresponding to the second English version) [4], and the International Union Against Cancer (UICC)/TNM classification system, which is in its sixth edition [5]. These staging systems are constantly evolving due to periodic validation studies. The aim of the present study was to evaluate the ability of these two systems to predict survival in light of the latest postoperative survival results and to propose improvements for a better prognostic stratification for gastric cancer.

An apparent difference between the JCGC and UICC systems lies in the way lymph node metastasis is evaluated. Whereas the JCGC system evaluates the extent of anatomical lymphatic spread, the UICC system takes into account only the number of positive nodes. Several previous studies evaluating which of these two systems is superior have been controversial [6-10]. The most recent surgical outcomes, based on the long-term followup of gastric cancer patients in 113 top Japanese hospitals, were reported according to the JCGC system [11]. However, the 5-year survival rates of the subgroups in each stage were not shown; thus, the report did not include validation of staging. The aim of the present study was to validate both the JCGC and UICC staging systems from a prognostic point of view and to discuss problems related to these staging systems for the clinical assessment of gastric cancer. 


\section{Patients and methods}

Our study of staging validation was designed as a retrospective study for a total of 1010 patients who underwent gastrectomy at Kitasato University Hospital between January 1, 1990, and March 31, 2002, and were followed for at least 5 years. Double cancers, surgical deaths, and deaths caused by other diseases were not included in the prognostic analysis. According to the JCGC, the stages of the 1010 gastric cancer patients were: IA $(n=453)$, IB $(n=185)$, II $(n=119)$, IIIA $(n=$ $75)$, IIIB $(n=51)$, and IV $(n=127)$. Clinical parameters in all patients were informative for the latest classifications, i.e., those in the thirteenth edition (second English edition) of the JCGC [4] and the sixth edition of the UICC/TNM staging system [5], including prognostic information on disease-specific death (Table 1). Eight hundred and eighty-three patients with stage IA to IIIB disease underwent R0 curative surgery, and patients with stage IV underwent gastric cancer resection, as previously described [12]. Of the 127 stage IV gastric cancer patients, R0 gastrectomy was performed in 37 , and $\mathrm{R} 1 / \mathrm{R} 2$ in 90 . The patients with $\mathrm{R} 1 / \mathrm{R} 2$ gastrectomy showed distant metastases $(n=7)$, peritoneal dissemination and/or positive cytology $(n=67)$, and/or lymph node metastasis of more than N3 $(n=67)$.

Staging was determined using both the JCGC and the UICC staging systems. In patients with multiple lesions in the stomach, the lesion which invaded the deepest layer of the stomach or which had the largest diameter when multiple lesions invaded the same layer was considered representative. Nodal status, including the number of metastatic nodes, was reviewed from pathology records. Metastasis was found histologically in 3562 lymph nodes (range, 0 to 61 metastatic nodes/ patient; average, 3.5 metastatic nodes/patient [including pN0 patients]), and at least 16 metastatic nodes were extracted in the individual cases.

Statistical computations were performed using the SAS software package StatView, version 5.0 (SAS Institute, Cary, NC, USA). A result was considered statistically significant when the $P$ value was less than $5 \%$ $(P<0.05)$. The time of follow-up was calculated from the surgery date. Disease-specific survival (DSS) was

Table 1. Distribution of pTNM factors in 1010 gastric cancer patients undergoing gastrectomy

\begin{tabular}{|c|c|c|}
\hline Parameters & No. of patients & Percentage \\
\hline \multicolumn{3}{|l|}{ pT factor (JCGC, UICC) } \\
\hline $\operatorname{Mucosa}(\mathrm{M}, \mathrm{pT} 1)$ & 296 & 29 \\
\hline Submucosa (SM, pT1) & 211 & 21 \\
\hline Muscularis propria (MP, pT2) & 94 & 9 \\
\hline Subserosa (SS, pT2) & 226 & 22 \\
\hline Serosa exposure (SE, pT3) & 151 & 15 \\
\hline Invasion of adjacent structure (SI, pT4) & 32 & 3 \\
\hline \multicolumn{3}{|l|}{ JCGC-pN factor } \\
\hline pNO & 628 & 62 \\
\hline $\mathrm{pN} 1$ & 184 & 18 \\
\hline $\mathrm{pN} 2$ & 136 & 13 \\
\hline pN3 & 51 & 5 \\
\hline $\mathrm{M}$ & 11 & 1 \\
\hline \multicolumn{3}{|l|}{ UICC-pN factor } \\
\hline $\mathrm{pN} 0$ & 628 & 62 \\
\hline pN1: $1-6$ nodes & 211 & 21 \\
\hline pN2: $7-15$ nodes & 66 & 7 \\
\hline pN3: 16 or more nodes & 45 & 4 \\
\hline Extraregional lymph node metastasis & 60 & 6 \\
\hline \multicolumn{3}{|l|}{ JCGC pStage } \\
\hline Stage IA & 453 & 45 \\
\hline Stage IB & 185 & 18 \\
\hline Stage II & 119 & 12 \\
\hline Stage IIIA & 75 & 7 \\
\hline Stage IIIB & 51 & 5 \\
\hline Stage IV & 127 & 13 \\
\hline \multicolumn{3}{|l|}{ UICC pStage } \\
\hline Stage IA & 453 & 45 \\
\hline Stage IB & 197 & 19 \\
\hline Stage II & 142 & 14 \\
\hline Stage IIIA & 64 & 6 \\
\hline Stage IIIB & 31 & 3 \\
\hline Stage IV & 123 & 12 \\
\hline
\end{tabular}


estimated according to the Kaplan-Meier method and results were compared using the log-rank test $[13,14]$.

\section{Results}

\section{Comparison of prognoses between the JCGC and UICC/TNM staging systems}

The characteristics of the 1010 gastric cancer patients included in this study are displayed in Table 1 . The prognosis of the 453 patients with stage IA disease was identical for both systems, and 5-year survival was $100 \%$. Figure $1 \mathrm{~A}-1$ shows the survival curves of the 557 gastric cancer patients with stage IB to IV disease according to the JCGC system. The 5-year survival rates were $85.8 \%$ for stage IB, $66.8 \%$ for stage II, $39.8 \%$ for stage IIIA, $25.4 \%$ for stage IIIB, and $4.7 \%$ for stage IV. The differences between these curves were statistically significant. The survival curves according to the UICC system are shown in Fig. 1A-2. The 5-year survival rates were $85.8 \%$ for stage IB, $60.8 \%$ for stage II, $30.8 \%$ for stage IIIA, $16.1 \%$ for stage IIIB, and $10.1 \%$ for stage IV. Significant differences were found between only some of the curves (stages IA and IB, $P<0.0001$, data not shown; stages IB and II, $P<0.0001$; stages II and IIIA, $P<0.0001$; stages IIIA and IIIB, $P=0.006$; stages IIIB and IV, no significant difference). The UICC system could not differentiate between the prognosis for patients with stage IIIB disease and that for patients with stage IV in our patient cohort.

All stage IA patients and $98 \%$ of stage IB patients according to the JCGC were also staged as IA and IB by the UICC. However, the agreement rates between the two systems at stages II, IIIA, IIIB, and IV were $84 \%, 51 \%, 24 \%$, and $64 \%$, respectively, indicating that advanced gastric cancer tended to be evaluated differently by the two systems. In total, 875 of the 1010 patients $(87 \%)$ were staged identically by the two systems, and their survival curves (Fig. 1B) were very similar to those of all patients staged by the JCGC system (Fig. 1A). In 134 patients (13\%), staging by the two systems disagreed. The survival curves of these 134 patients according to the two staging system are shown in Fig. 1C. The JCGC system maintained a clear stratification between the stages, while the UICC system failed to do so.

All the patients with stage IB disease according to the UICC had T1 (invasion of the submucosa [SM]) cancers with Japanese N2 nodal metastasis (thus, JCGC stage II) but with six or fewer nodal metastases (thus, UICC N1). A considerable number of these patients did not survive because of cancer recurrence, suggesting that the JCGC N2 classification may be prognostically significant in early gastric cancer, as we had previously reported [15]. On the other hand, of the 123 patients with stage IV according to the UICC system, 42 had undergone a curative operation, and the stages in these 42 patients corresponded to stage IB $(n=1)$, stage II $(n=2)$, and stage III $(n=39)$ according to the JCGC system. These patients showed a relatively good prognosis, and all 3 patients with JCGC stage IB or II disease survived for more than 5 years despite each having more than 16 metastatic lymph nodes. Of the 36 patients with stage IV designated exclusively by the JCGC staging system, the UICC stages were: stage II $(n=2)$, stage IIIA $(n=10)$, and stage IIIB $(n=24)$. The reason for stage IV classification by the JCGC system exclusively was either anatomical node metastasis $(n=23)$ and/or positive cytology $(n=15)$ ("anatomical node metastasis" included perigastric nodal metastasis far from the primary tumor [Japanese N3, but UICC N1 or N2] such as No. 5 or 6 nodal metastases from the upper gastric tumor $[n=15]$, No. 2 or 4 sa nodal metastasis from a middle gastric body [ $n=5]$, or No. 2, 4sa, or 4sb nodal metastasis from a lower gastric tumor $[n=3]$ ). The 23 patients with JCGC N3 lymph node metastasis within perigastric nodes showed dismal prognoses (only 1 patient survived for more than 5 years).

\section{Prognostic heterogeneity in JCGC stages II and IIIA}

We examined the prognostic stratification according to depth of invasion within each stage, using the JCGC staging system (Fig. 2). Stage IB consists of cases involving invasion of the mucosa (M), submucosa (SM), muscularis propria (MP), and subserosa (SS). There were only 2 patients with $\mathrm{M}$ cancer with $\mathrm{pN} 1$ metastasis, and both survived for more than 5 years. Patients with MP cancer with no metastasis showed a prognosis similar to that for patients with SM cancer with $\mathrm{pN} 1$ metastasis. On the other hand, patients with MP cancer with no metastasis appeared to show a better prognosis than patients with SS cancer with no metastasis, but the difference was not statistically significant (Fig. 2A; $P=$ 0.11). In stage II, which consists of SM, MP, SS, and serosal exposure (SE), prognostic heterogeneity was recognized. In this stage, there were two distinct prognostic groups; SM/MP and SS/SE (Fig. 2B; $P=0.027$ ). Stage IIIA, which consists of MP, SS, SE, and invasion to a adjacent structure (SI), was the most problematic stage for exhibiting prognostic stratification according to depth of invasion (Fig. 2C). The most remarkable finding was that patients with MP cancer were all alive, even though their number was very small (5 of the 1010 patients). The second outstanding finding was that SI cancer showed a relatively better prognosis than expected. Finally, in both stages IIIB and IV, there was no heterogeneity of prognosis according to depth of invasion (Fig. 2C, D). 
(A) (1) JCGC stage

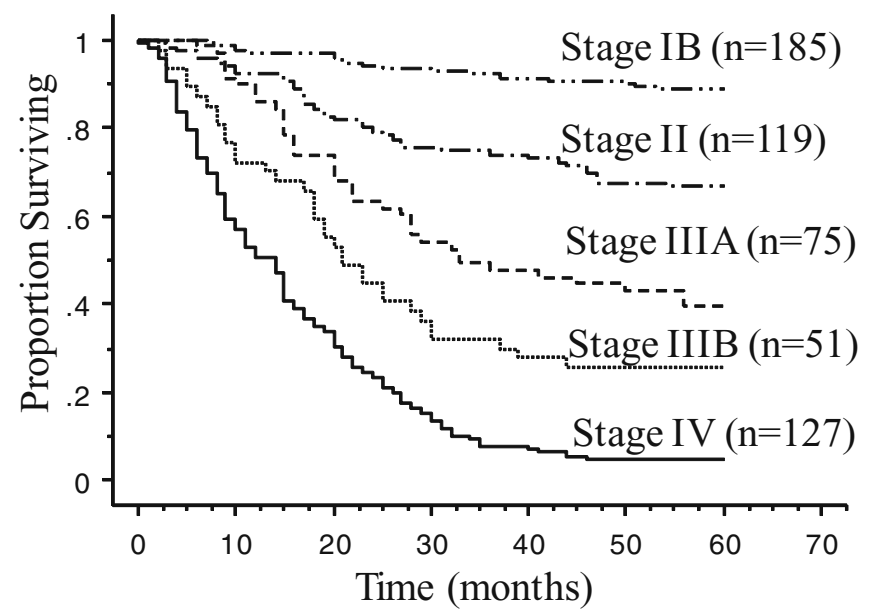

(2) UICC stage

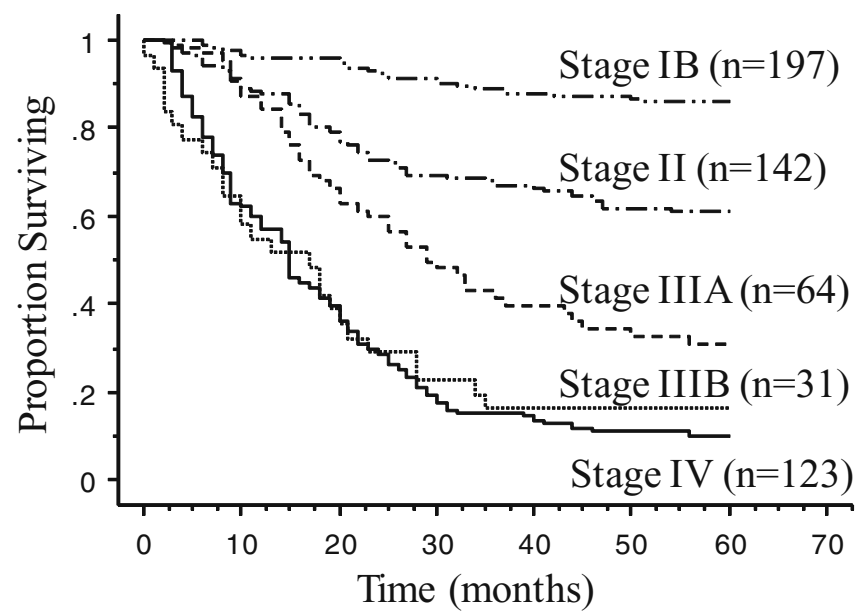

(B) Identical Case Analysis

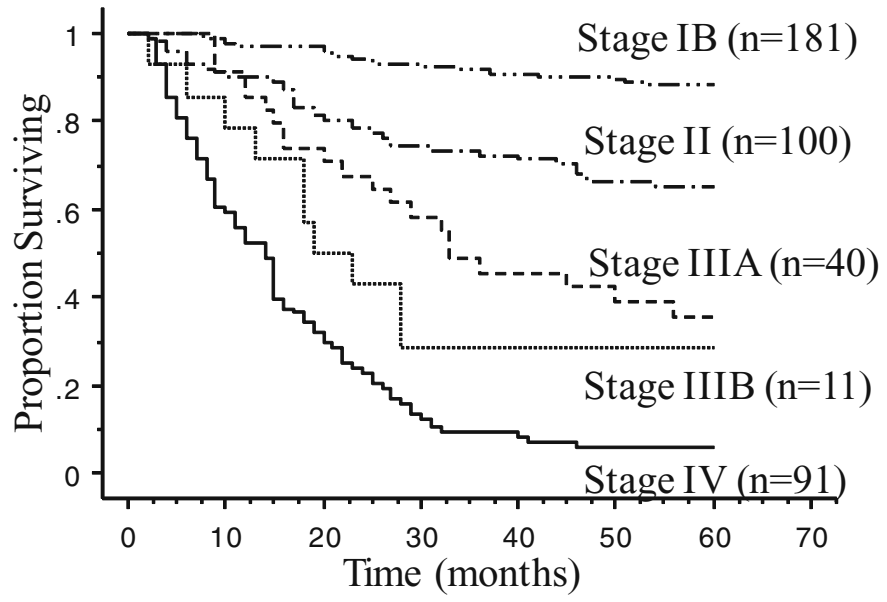

(C) Different Case Analysis

(1) JCGC stage

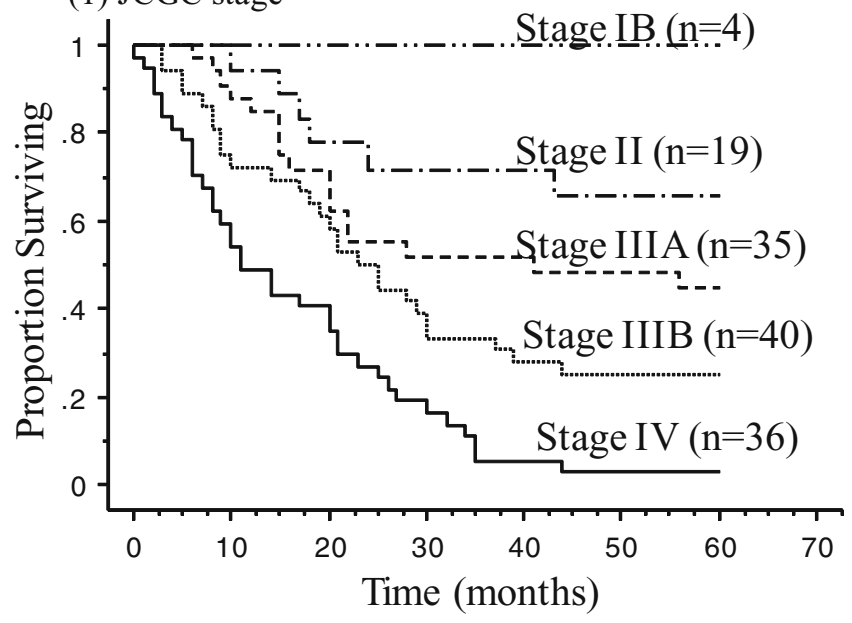

(2) UICC stage

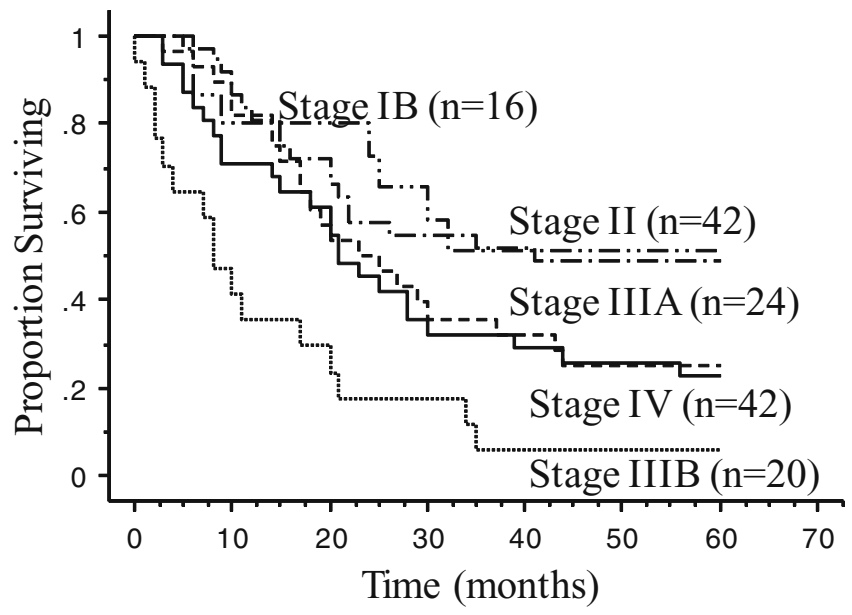

Fig. 1A-C. Prognostic comparison of the Japanese classification of gastric cancer (JCGC) and International Union Against Cancer (UICC)/TNM staging systems in 1010 gastric cancer patients. A Disease-specific survival of 1010 gastric cancer patients after gastrectomy, by Kaplan-Meier analysis, according to: (1) JCGC and (2) UICC staging (stage IA not shown). The UICC system showed inferior prognostic curves, except in stage IV. B Four hundred and twenty-three patients were identically evaluated by both systems, and the survival curve was similar to that given by the JCGC system alone (stage IA not shown). C One hundred and thirty-four patients were differently evaluated by the two systems, and the survival curve of the (1) exclusive JCGC system was similar to that generated by the JCGC system, with excellent stratification for survival (stage IA not shown). However, the survival curve generated according to the (2) exclusive UICC system did not show good prognostic stratification for survival 
(A) Stage IB

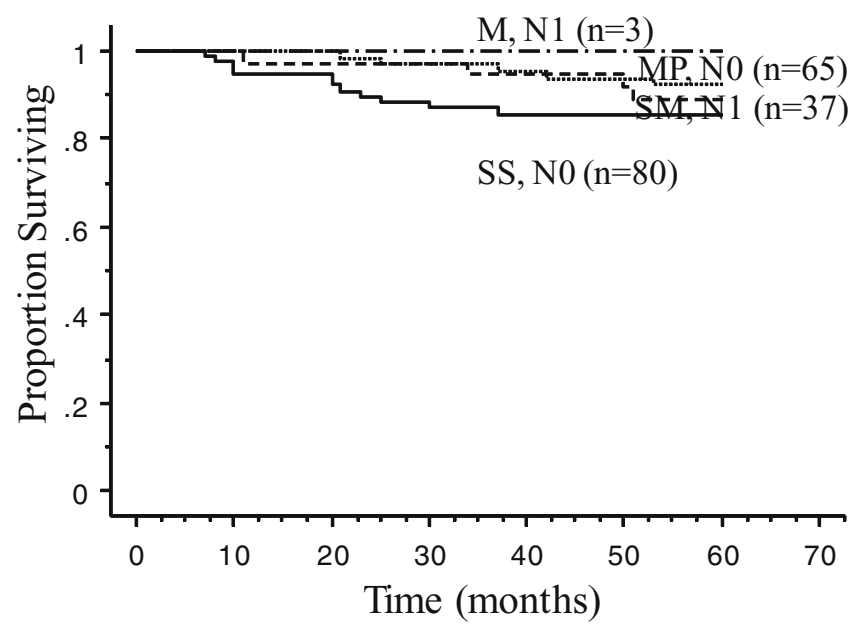

(B) Stage II

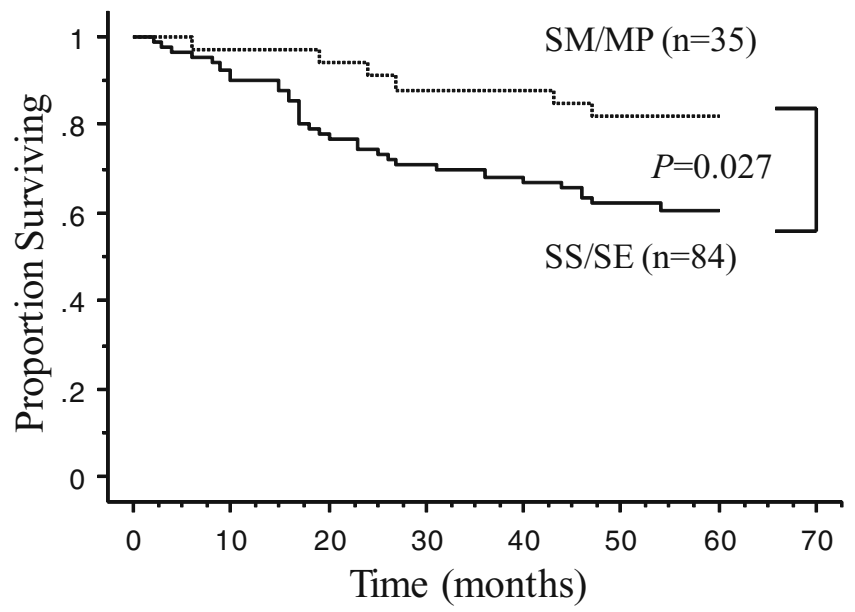

Fig. 2A-D. Prognostic analysis according to depth of invasion within JCGC stages by logrank test evaluating Kaplan-Meier survival curves. Disease-specific survival by Kaplan-Meier analysis of A, stage IB; $\mathbf{B}$, stage II; C, stage III, separately analyzed for stages IIIA (1) and IIIB (2); and D, stage IV. Note that stage II and stage IIIA were heterogeneous in prognosis even within the same stage
(C) (1) Stage IIIA

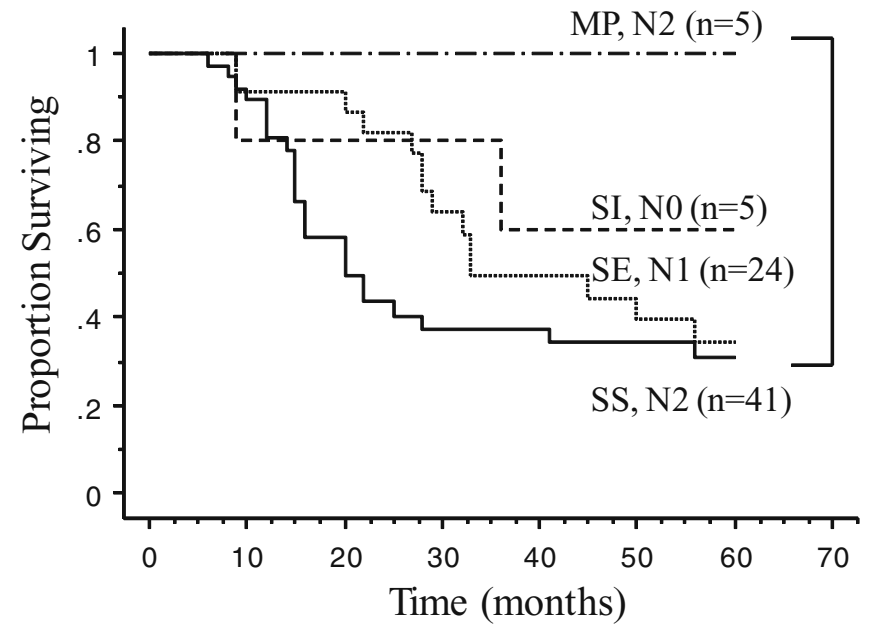

(2) Stage IIIB

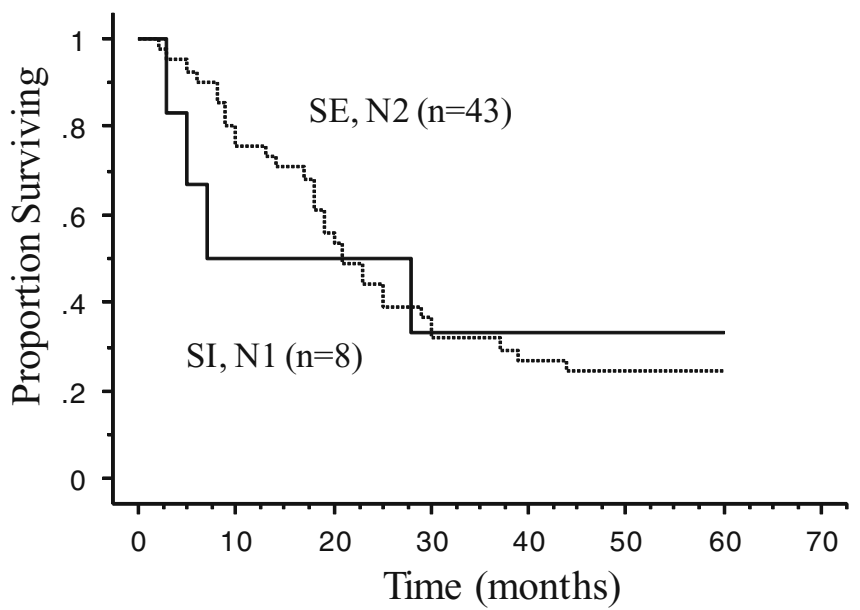

(D) Stage IV

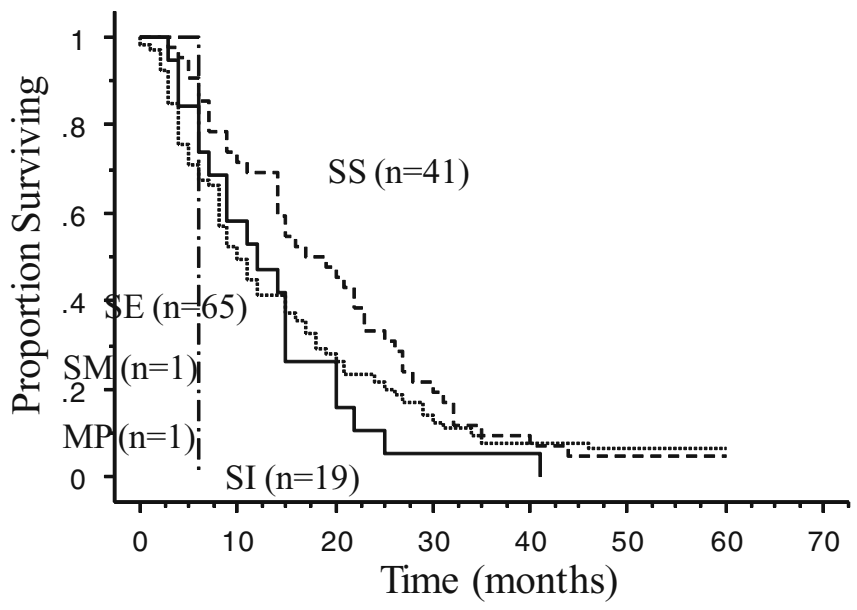


Proposal for novel JCGC staging system in gastric cancer

From our current analysis of the prognostic value of the current staging systems for gastric cancer, we propose novel subclassifications for the JCGC staging system, as shown in Fig. 3A. This novel staging system would include the following three changes: (1) MP cancer should be categorized as stage IB irrespective of lymph node metastasis status, (2) SI cancer with no metastasis should be categorized as stage II, and (3) there should be no subgroups within stage III. Using this system, we obtained clearer prognostic stratification according to stage by Kaplan-Meier survival analysis (Fig. 3B; stages
IA and IB; $P<0.0001$, data not shown; stages IB and II; $P<0.0001$; stages II and III; $P<0.0001$; stages III and IV; $P<0.0001$ ), in which homogeneous prognostic distribution was attained. The proposed staging system, combined with TN factors, is shown in Fig. 3C.

\section{Discussion}

Our analysis revealed that gastric cancer prognosis could be better stratified according to depth of invasion, classified into three groups, M/SM/MP, SS, and SE/SI (Fig. 4); this classification also, in turn, supports the
(A)

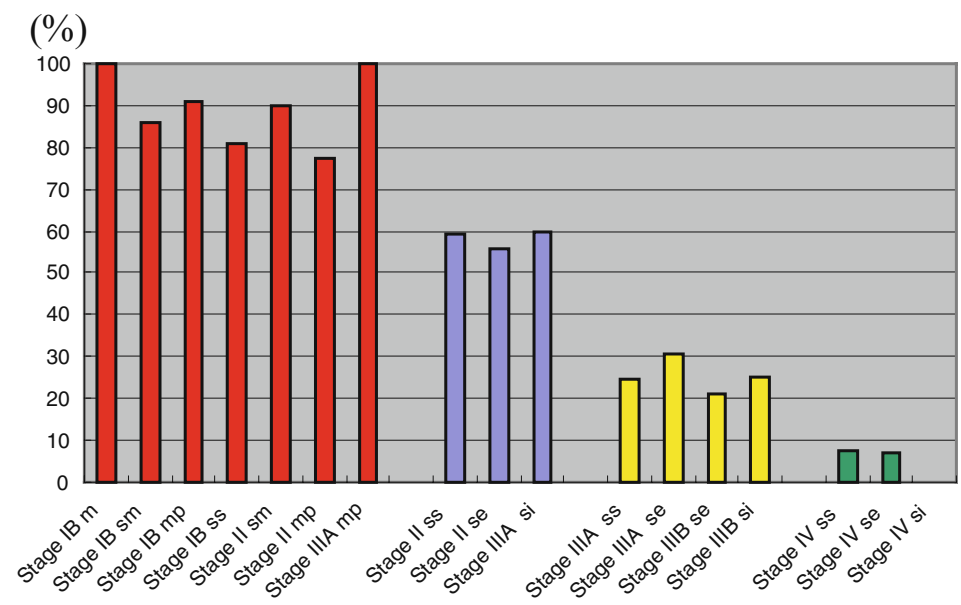

(B)

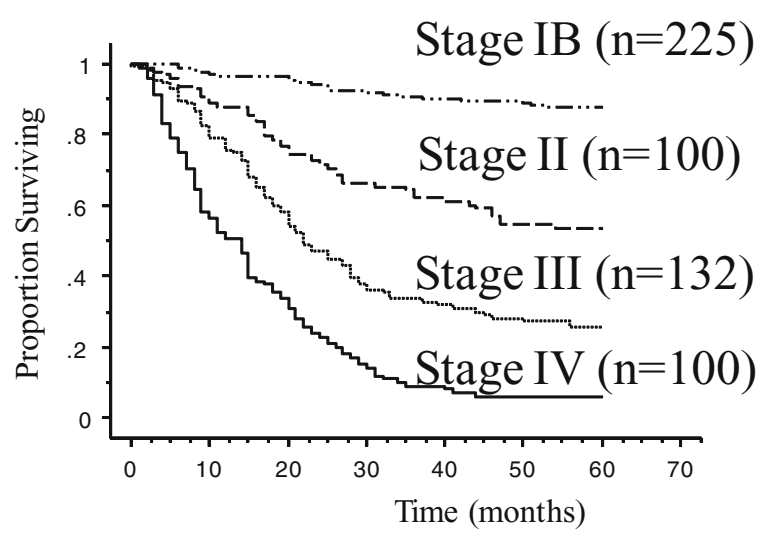

Fig. 3A-C. Proposed novel staging system after validation of the JCGC system. A Gastric cancer can be separated into four distinct prognostic groups. Note, survival rates according to depth of invasion within each stage showed homogeneous distribution in the following categories: best prognosis (red bars), better prognosis (blue bars), worse prognosis (yellow bars), and worst prognosis (green bars). B Our proposed novel staging system showed clearer prognostic stratification than
(C)

\begin{tabular}{|c|c|c|c|c|}
\hline & No & N1 & N2 & N3 \\
\hline$M$ & IA & IB & 2 & 4 \\
\hline SM & $\mathrm{IA}$ & IB & 2 & 4 \\
\hline $\mathrm{MP}$ & IB & 2 & $3 \mathrm{~A}$ & 4 \\
\hline SS & IB & 2 & $3 \mathrm{~A}$ & 4 \\
\hline SE & 2 & $3 A$ & $3 B$ & 4 \\
\hline $\mathrm{SI}$ & $3 A$ & $3 B$ & 4 & 4 \\
\hline & No & N1 & $\mathrm{N} 2$ & N3 \\
\hline$M$ & IA & IB & IB & 4 \\
\hline SM & IA & IB & IB & 4 \\
\hline MP & IB & IB & IB & 4 \\
\hline SS & IB & 2 & 3 & 4 \\
\hline SE & 2 & 3 & 3 & 4 \\
\hline $\mathrm{SI}$ & 2 & 3 & 4 & 4 \\
\hline
\end{tabular}

the present system. A significant difference was found between the representative curves $(\mathrm{P}<0.0001)$, and the number distribution was also appropriate. $\mathbf{C}$ The proposed novel JCGC system would take into account a combination of both depth of invasion and lymph node status. $M$, Invasion of the mucosa; $S M$, invasion of the submucosa; $M P$, invasion of the muscularis propria; $S S$, invasion of the subserosa; $S E$, serosal exposure; $S I$, invasion to adjacent structure 


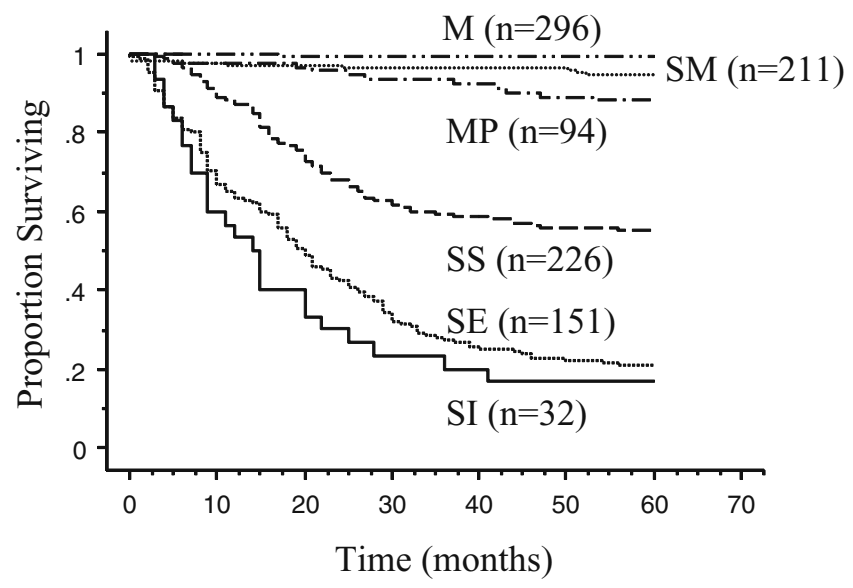

Fig. 4. Gastric cancer consists of three distinct prognostic groups classified according to depth of invasion

notion that MP cancer may be categorized as an earlyphase cancer rather than being placed in the same category as SS cancer. Currently, both the JCGC and UICC staging systems assign MP and SS cancers to the same T group, although the latest UICC staging system subdivided the pT2 category into pT2a (MP) and pT2b (SS). Invasion beyond the MP may be a critical point in differentiating between early and advanced cancers, as seen in Fig. 4. In 2004, Ishigami et al. [16] classified MP cancers into MP1, defined as invasion of the superficial layers, and MP2, invasion of the deeper layers, and revealed that the incidence of lymph node metastasis and the 5-year survival in patients with MP1 gastric cancer were similar to those in patients with SM gastric cancer. On the other hand, patients with MP2 cancers showed more macroscopic signs of advanced cancers, a higher frequency of lymph node metastasis, a lower rate of operative cure, and marginally shorter survival than those with MP1 cancers [16]. These findings suggest that the entity MP cancer is rather close to early gastric cancer. Our MP patients showed an excellent prognosis, with a DSS of about $90 \%$, which is much better than that demonstrated by the nationwide registry of gastric cancer (NWR-G; 78.4\%) in 1991 [11]. The reason for this better result may reflect the inclusion of more MP1 cancers in our study than in the NWR-G data; thus, consideration of MP1 vs MP2 may be helpful for more accurate staging, and these categories should be validated in the near future.

In the present study, our data suggest that, from a prognostic point of view, MP cancer may be assigned to stage IB irrespective of lymph node metastasis. However, the number of patients was too small to make a firm conclusion, and further validation remains necessary. The 5-year survival rates of stage IB and stage II patients in our study were $85.8 \%$ and $66.8 \%$, respectively, a result which is similar to those reported in the NWR-G (84.7\% and $69.1 \%$ ) [11], suggesting that our treatment outcome is consistent with the overall average outcome for Japanese patients with stage IB and stage II gastric cancer. On the other hand, the prognosis (5-year survival rate) was worse for stage IIIA patients in our study $(39.8 \%)$ than in the NWR-G $(50.1 \%)$. The distribution of depth of invasion in our pT2/T3 patients (MP:SS:SE $=20: 48: 32$ ) was different from that in the NWR-G data $(23: 32: 45)$. Thus, the pathological criteria used to separate SS and SE at our institution may have been biased, thereby causing the great difference in prognosis between MP and SS cancers. An alternative explanation is that our data were based on the DSS, while the NWR$\mathrm{G}$ data were based on overall survival.

In this study, we also pointed out the excellent features of the JCGC staging system. The three important comparative studies so far of the JCGC and UICC staging systems have demonstrated the superiority of the UICC system for evaluating the contribution of lymph node metastasis status to prognosis [6-8]. These reports showed that the UICC classification of lymph node metastasis by number could further divide the prognosis of the identical JCGC stage patients between UICC $\mathrm{pN} 1$ and $\mathrm{pN} 2$, but not vice versa [6,7]. We performed survival analysis for stage II and stage III patients by using UICC $\mathrm{pN} 1$ and $\mathrm{pN} 2$, and the prognosis was clearly stratified by the number of lymph node metastases, as previously reported $(P=0.02$ and $P=$ 0.0004 , respectively) $[6,7]$. In our present study, the JCGC and UICC staging systems correlated well with each other, suggesting that the anatomical extent of lymph node metastasis is closely associated with the number of metastatic nodes, and that the dissociative (different) cases were characterized by the inferiority of the UICC system for prognostic stratification for worse prognosis in stage IB and better prognosis in stage IV than expected (Fig. 1C [UICC]). As stated earlier in the Results section, we demonstrated the superiority of the JCGC system over the UICC system in predicting the prognosis of JCGC N2 disease in early gastric cancer and for consideration of perigastric JCGC N3 nodules and/or cytology factors in advanced gastric cancers. The most intriguing issue is that these findings involve anatomical considerations, for which the JCGC system is most suited.

In conclusion, the UICC $\mathrm{pN}$ classification is a definitive prognostic factor within the same JCGC stage, as previously reported $[6,7]$. Thus, for a more consistent stratification of patient prognosis, it would be beneficial to consider the number of involved nodes, in combination with the anatomical considerations given by the JCGC staging system. The present JCGC staging system should be refined by further validation, especially for the pT2 categories. 


\section{References}

1. Desai AM, Pareek M, Nightingale PG, Fielding JW. Improving outcomes in gastric cancer over 20 years. Gastric Cancer 2004;7:196-203.

2. Crew KD. Epidemiology of gastric cancer. World J Surg 2006; 12:354-62.

3. Yokota T, Ishiyama S, Saito T, Teshima S, Shimotsuma M, Yamaguchi $\mathrm{H}$. Treatment strategy of limited surgery in the treatment guidelines for gastric cancer in Japan. Lancet Oncol 2003;4: 423-8.

4. Japanese Gastric Cancer Association. Japanese classification of gastric carcinoma - 2nd English edition -. Gastric Cancer 1998;1: $10-24$.

5. Sobin $\mathrm{LH}$, Witteking $\mathrm{Ch}$, editors. International Union Against Cancer TNM classification of malignant tumours. 6th ed. New York: Wiley-Liss; 2002.

6. Ichikura T, Tomimatsu S, Uefuji K, Kimura M, Uchida T, Morita D, et al. Evaluation of the New American Joint Committee on Cancer/International Union against cancer classification of lymph node metastasis from gastric carcinoma in comparison with the Japanese classification. Cancer 1999;86:553-8.

7. Fujii K, Isozaki H, Okajima K, Nomura E, Niki M, Sako S, et al. Clinical evaluation of lymph node metastasis in gastric cancer defined by the fifth edition of the TNM classification in comparison with the Japanese system. Br J Surg 1999;86:685-9.

8. Hayashi H, Ochiai T, Suzuki T, Shimada H, Hori S, Takeda A, et al. Superiority of a new UICC-TNM staging system for gastric carcinoma. Surgery 2000;127:129-35.
9. Nio Y, Yamasawa K, Yamaguchi K, Itakura M, Omori H, Koike M, et al. Problems in the N-classification of the new 1997 UICC TNM stage classification for gastric cancer: an analyisis of over 10 years' outcome of Japanese patients. Anticancer Res 2003;23: 697-705.

10. Ikeguchi M, Murakami D, Kanaji S, Ohro S, Maeta Y, Yamaguchi $\mathrm{K}$, et al. Lymph node metastasis of gastric cancer: comparison of Union International Contra Cancer and Japanese systems. ANZ J Surg 2004;74:852-4.

11. Japanese Gastric Cancer Association Registration Committee, Maruyama K, Kaminishi M, Hayashi K, Isobe Y, Honda I, et al. Gastric cancer treated in 1991 in Japan: data analysis of nationwide registry. Gastric Cancer 2006;9:51-66.

12. Yamashita K, Sakuramoto S, Kikuchi S, Katada N, Kobayashi N, Watanabe M. Surgical resection of stage IV gastric cancer and prognosis. Anticancer Res 2007;27:4381-6.

13. Kaplan EL, Meier P. Nonparametric estimation from incomplete observations. J Am Stat Assoc 1958;53:457-81.

14. Cox DR. Regression models and life-tables. J R Stat Soc (B) 1972:34:187-220.

15. Kikuchi S, Sato M, Katada N, Sakuramoto S, Kobayashi N, Shimao H, et al. Surgical outcome of node-positive early gastric cancer with particular reference to nodal status. Anticancer Res 2000;20:3695-700.

16. Ishigami S, Natsugoe S, Miyazono F, Hata Y, Uenosono Y, Sumikura S, et al. Clinical merit of subdividing gastric cancer according to invasion of the muscularis propria. Hepatogastroenterology 2004;51:869-71. 